Article

\title{
Structural, Electronic, Magnetic, Mechanic and Thermodynamic Properties of the Inverse Heusler Alloy $\mathrm{Ti}_{2} \mathrm{NiIn}$ Under Pressure
}

\author{
Tie Yang * (D), Jieting Cao and Xiaotian Wang * \\ School of Physical Science and Technology, Southwest University, Chongqing 400715, China; \\ 13308348369@163.com \\ * Correspondence: yangtie@swu.edu.cn (T.Y.); xiaotianwang@swu.edu.cn (X.W.)
}

Received: 10 October 2018; Accepted: 13 November 2018; Published: 15 November 2018

\begin{abstract}
Structural, electronic, magnetic and mechanic properties of the inverse Heusler alloy $\mathrm{Ti}_{2} \mathrm{NiIn}$ under different pressure are systematically studied with density functional theory (DFT). The equilibrium lattice constant and electronic band structure at null pressure are obtained to be consistent with previous work. Under currently applied static pressure from $0 \mathrm{GPa}$ to $50 \mathrm{GPa}$, it is found that the half-metallicity of the material is maintained and the total magnetic moment $\left(\mathrm{M}_{\mathrm{t}}\right)$ is kept at $3 \mu_{B}$, which obeys the Slater-Pauling rule, $M_{t}=Z_{t}-18$, where $Z_{t}$ is the total number of valence electrons. Besides, the effect of the tetragonal distortion was studied and it is found that the magnetic property of $\mathrm{Ti}_{2} \mathrm{NiIn}$ is almost unchanged. Several mechanical parameters are calculated including three elastic constants, bulk modulus B, Young's modulus E, and shear modulus $\mathrm{S}$ and the mechanical stability is examined accordingly. Furthermore, the thermodynamic properties, such as the heat capacity $C_{V}$, the thermal expansion coefficient $\alpha$, the Grüneisen constant $\gamma$ and the Debye temperature $\Theta_{\mathrm{D}}$, are computed by using the quasi-harmonic Debye model within the same pressure range at a series of temperature from 0 to $1500 \mathrm{~K}$. This theoretical study provides detailed information about the inverse Heusler compound $\mathrm{Ti}_{2} \mathrm{NiIn}$ from different aspects and can further lead some insight on the application of this material.
\end{abstract}

Keywords: heusler compound; density functional theory; first-principle calculation

\section{Introduction}

During the last decade, half-metallic materials (HMMs) have attracted tremendous attention and become of great interest because they can provide novel functionalities for spintronic [1] and magneto-electronic materials [2,3]. The electronic structure of HMMs shows metallic behavior with no band gap for one channel and semiconducting behavior with band gap in the other channel, leading to a possible $100 \%$ spin polarization of electrons near the Fermi energy level [4,5]. Among these materials, the Heusler alloy family stands out because of its high Curie temperature and tunable physical properties [6-8]. Besides, the Heusler materials have comparable crystal structure with zinc-blende-type and rock-salt-type semiconductors [9-11], making them suitable for the spin injection source of semiconductors. The first-principles calculations have been successfully and extensively applied to study the physical properties of Heusler alloys, including electronic property, magnetic property, mechanical property, etc. Many Heusler compounds have been predicted theoretically to be half-metal and many experiments also have been carried out to establish this half-metallicity [12-14].

Heusler alloys are commonly represented by a generic formula $X_{2} Y Z$ [15-17], in which $X$ and $\mathrm{Y}$ stand for transition metal elements and Z for s-p element. Normally, the Heusler compounds crystallize in a highly ordered cubic structure either in $\mathrm{Cu}_{2} \mathrm{MnAl}$-type or $\mathrm{Hg}_{2} \mathrm{CuTi}$-type, and there 
is half metallic behavior found in both types. We should point out here that $\mathrm{Hg}_{2}$ CuTi-type full Heusler alloys have been widely studied and a number of this type alloys exhibit half-metallic properties [18-20], and also, many of $\mathrm{Hg}_{2} \mathrm{CuTi}$ alloys obey the well-known Slater-Pauling rule [21-23]. More important, some $\mathrm{Hg}_{2} \mathrm{CuTi}$-type $\mathrm{Ti}_{2}$-based full Heusler alloys have been predicted to be spin-gapless semiconductors [18,24] or magnetic Weyl semimetal [25]. Thus, Ti ${ }_{2}$-based Heusler becomes of great interest nowadays.

Several Ti ${ }_{2} \mathrm{Ni}$-based full Heusler alloys have been theoretically studied, such as Feng et al. $[26,27]$ demonstrated $\mathrm{Ti}_{2} \mathrm{NiAl}$ and $\mathrm{Ti}_{2} \mathrm{NiIn}$ being half-metal with complete spin polarization around the Fermi level; Wei et al. [19] and Boudali et al. [28] performed a series of studies on the half-metallic, structural, mechanical properties of $\mathrm{Ti}_{2} \mathrm{NiZ}(\mathrm{Z}=\mathrm{Al}, \mathrm{Ga}, \mathrm{In})$; Wei et al. [29] and Feng et al. [27] made detailed analysis of the electronic and magnetic properties of defects in $\mathrm{Ti}_{2} \mathrm{NiIn}$ and also examined the stability and thermodynamic properties on $\mathrm{Ti}_{2} \mathrm{NiIn}$; Wen et al. concentrated on the mechanical and acoustic properties of $\mathrm{Ti}_{2} \mathrm{NiZ}(\mathrm{Z}=\mathrm{Al}, \mathrm{Ga}, \mathrm{In})$ Heusler alloys. In this work, we focus on $\mathrm{Ti}_{2} \mathrm{NiIn}$ compound and carry out a systematic study on its strain condition in terms of the pressure and temperature dependence of its structural, electronic, magnetic and mechanical properties based on first-principles calculation and the quasi-harmonic Debye model. Different from previous studies, we further enlarge the applied static pressure to $50 \mathrm{GPa}$ and elevate the temperature limit to $1500 \mathrm{~K}$ and also correlated the different lattice constants with pressure by geometry optimization. Thus, we can reveal the effect of external pressure to better understand the real condition. Besides, we also discuss the tetragonal distortion and its impact on the half-metallic, electronic and magnetic properties of $\mathrm{Ti}_{2} \mathrm{NiIn}$.

\section{Computational Details}

\subsection{Crystal Structure and Equilibrium Lattice}

The cubic Heusler compounds $\mathrm{X}_{2} \mathrm{YZ}$ commonly have $\mathrm{Cu}_{2} \mathrm{MnAl}$-type structure or $\mathrm{Hg}_{2} \mathrm{CuTi-type}$ structure. The former one is also known as $\mathrm{L}_{21}$ structure and the latter on as $\mathrm{XA}$ structure or inverse structure. In either one, the basic crystal structure is based on four interpenetrating face-centered-cubic (FCC) sublattices defined by Wyckoff coordinates as A $(0,0,0), B(0.25,0.25$, $0.25), C(0.5,0.5,0.5)$ and $\mathrm{D}(0.75,0.75,0.75)$. For the currently studied $\mathrm{Ti}_{2} \mathrm{NiIn}$ alloy, we adopt the inverse structure and the position sites $\mathrm{A}, \mathrm{B}, \mathrm{C}$ and $\mathrm{D}$ are occupied by $\mathrm{Ti}, \mathrm{Ti}, \mathrm{Ni}$ and $\mathrm{In}$ atoms, accordingly, see the inset crystal structure in Figure 1. We have performed first-principles calculations using the pseudo-potential plane-wave methods based on density functional theory (DFT) [30] as implemented in the CASTEP codes [31]. The exchange-correlation functional scheme is selected as the Perdew-Burke-Ernzerhof (PBE) functional within the generalized gradient approximation (GGA) [32]. The ultrasoft pseudopotential is applied to describe the interaction between ions and electrons. After the convergence test, the following parameters are set: $500 \mathrm{eV}$ plane-wave cutoff energy, $5 \times 10^{-6} \mathrm{eV}$ self-consistent field tolerance and $12 \times 12 \times 12 \mathrm{k}$-point mesh.

To determine the equilibrium lattice constant of $\mathrm{Ti}_{2} \mathrm{NiIn}$, we first compute the total energy at different lattice constants and the result is shown in Figure 1. By polynomial fitting and total energy minimization, we successfully obtain the equilibrium lattice constant, $6.42 \AA$, which is in very good agreement with other studies [27-29,33,34]. 


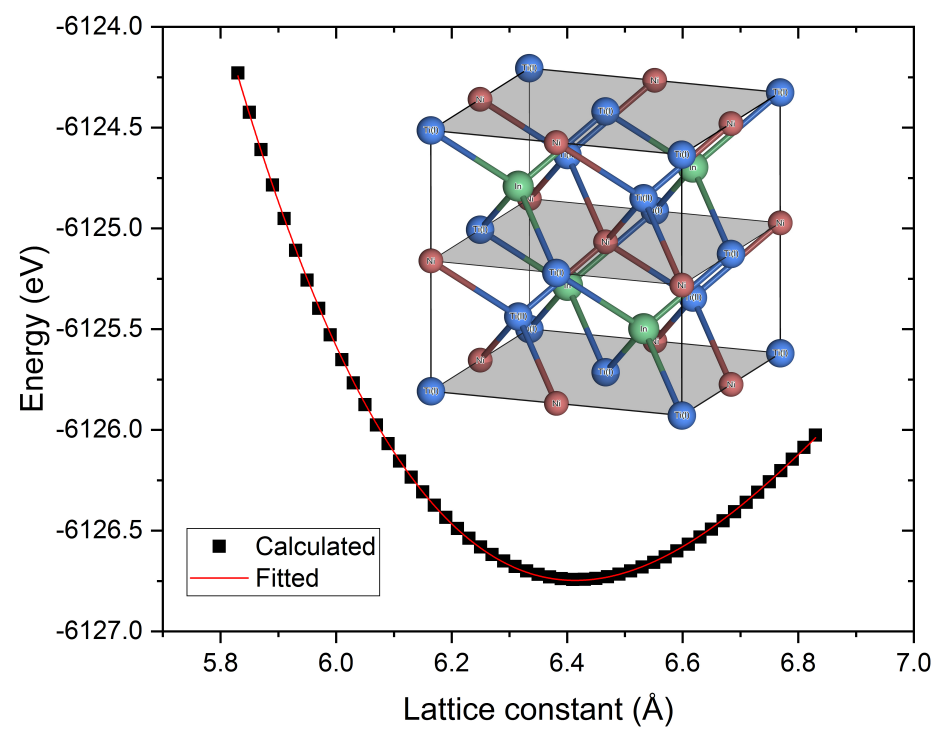

Figure 1. The calculated total energy of $\mathrm{Ti}_{2} \mathrm{NiIn}$ compound with respect to different lattice constant. Inset is the corresponding crystal structure.

\subsection{Electronic and Magnetic Properties}

Based on the obtained equilibrium lattice, we can calculate the electronic structure and magnetic properties of $\mathrm{Ti}_{2} \mathrm{NiIn}$ with PBE-GGA and ultrasoft pseudopotential to describe the exchange-correlation functional and the interaction between ions and electrons. Furthermore, for the application of external pressure, we performed structural optimization with the Broyden-Fletcher-Goldfarb-Shanno (BFGS) minimization [35]. The converge parameters for this structure optimization are set as follows: the energy change per atom is less than $5 \times 10^{-6} \mathrm{eV}$; the forces on atoms are less than $0.01 \mathrm{eV} / \AA$ and all the stress components are less than $0.02 \mathrm{GPa}$. Figure 2 shows the lattice constants and total energies under different pressure. With pressure increase, the lattice decreases yet the total energy increases. After the structure optimization under different pressure, the electronic and magnetic behaviors at different pressure were calculated accordingly.

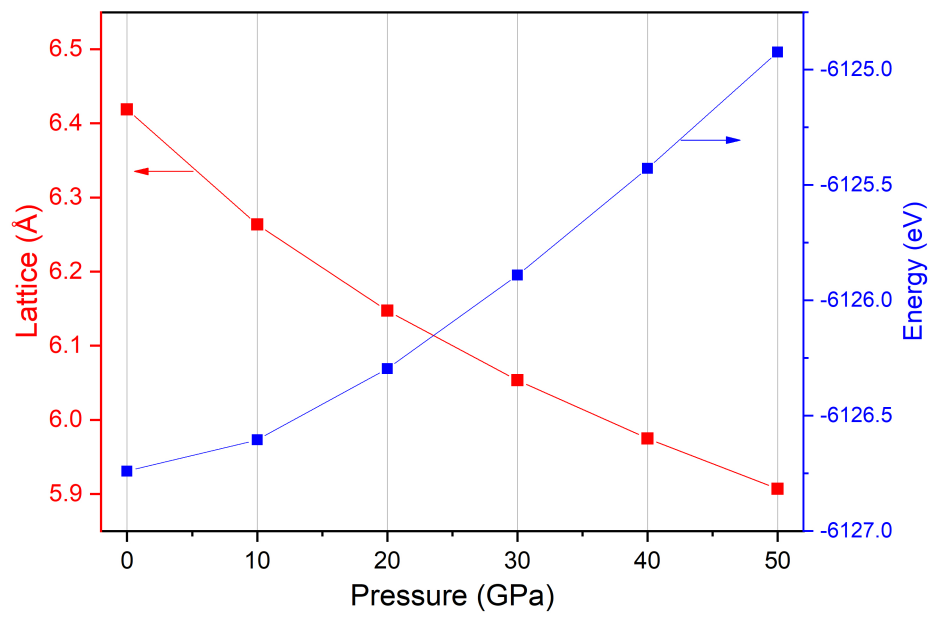

Figure 2. The calculated lattice constant and total energy of $\mathrm{Ti}_{2} \mathrm{NiIn}$ compound under different external pressure. $0 \mathrm{GPa}$ refers to the equilibrium condition. 


\subsection{Mechanical Properties}

For the mechanical properties of $\mathrm{Ti}_{2} \mathrm{NiIn}$, we first study its elastic constants and they are obtained by the stress-strain method [36]. For a cubic structure, there are three independent elastic constants, namely, $C_{11}, C_{12}$ and $C_{14}$, in which $C_{11}$ represents the elasticity in length and the other two characterize the elasticity in shape. In addition, the mechanical stability for cubic structure crystal can be judged by the following generalized Born-Huang elastic stability criteria [37,38]:

$$
\mathrm{C}_{11}+2 \mathrm{C}_{12}>0, \quad \mathrm{C}_{11}-\mathrm{C}_{12}>0, \quad \mathrm{C}_{44}>0
$$

With these three elastic constants we can further calculated other mechanical properties by the Voigt-Reuss-Hill (VRH) approximation [39]. The bulk modulus B and the shear modulus G generally describe the resistance to volume and shape variation and can be given by

$$
\mathrm{B}=\frac{\mathrm{B}_{\mathrm{R}}+\mathrm{B}_{\mathrm{V}}}{2}, \quad \mathrm{G}=\frac{\mathrm{G}_{\mathrm{R}}+\mathrm{G}_{\mathrm{V}}}{2}
$$

where $B_{R}\left(B_{V}\right)$ and $G_{R}\left(G_{V}\right)$ represent the lower (upper) limit for polycrystalline crystal at the Reuss (Voigt) boundary. For a cubic crystal, they have the following relations with the elastic constants [40]:

$$
B_{R}=B_{V}=\frac{C_{11}+2 C_{12}}{3}, \quad G_{V}=\frac{C_{11}-C_{12}+3 C_{44}}{5}, \quad G_{R}=\frac{5 C_{44}\left(C_{11}-C_{11}\right)}{4 C_{44}+3\left(C_{11}-C_{12}\right)}
$$

Then, the Young's modulus E and Poisson's ratio $\nu$ can be determined from B and G by the following formula [41]:

$$
\mathrm{E}=\frac{9 \mathrm{~GB}}{3 \mathrm{~B}+\mathrm{G}}, \quad v=\frac{3 \mathrm{~B}-2 \mathrm{G}}{2(3 \mathrm{~B}+\mathrm{G})}
$$

\subsection{Thermodynamic Properties}

In order to understand the specific behavior of $\mathrm{Ti}_{2} \mathrm{NiIn}$ compound under severe constraints, we further studied its thermodynamic properties and their dependencies on temperature and pressure by the quasi-harmonic Debye model [42-44]. The studied temperature range is from 0 to $1500 \mathrm{~K}$ and pressure range from 0 to $50 \mathrm{GPa}$. In the quasi-harmonic Debye model, the non-equilibrium Gibbs free energy is expressed by

$$
\mathrm{G}^{*}(\mathrm{~V} ; \mathrm{P}, \mathrm{T})=\mathrm{E}(\mathrm{V})+\mathrm{PV}+\mathrm{A}_{\mathrm{vib}}\left(\Theta_{\mathrm{D}}(\mathrm{V}) ; \mathrm{T}\right)
$$

where $\mathrm{E}(\mathrm{V})$ is the total energy per unit cell of $\mathrm{Ti}_{2} \mathrm{NiIn}$, PV represents the constant hydrostatic pressure condition, $\Theta_{\mathrm{D}}(\mathrm{V})$ corresponds the Debye temperature and $\mathrm{A}_{\mathrm{vib}}$ stands for the vibrational Helmholtz free energy, which can be expressed as follow using the Debye model of the phonon density:

$$
\mathrm{A}_{\mathrm{vib}}\left(\Theta_{\mathrm{D}} ; \mathrm{T}\right)=\mathrm{nk}_{\mathrm{B}} \mathrm{T}\left[\frac{9}{8} \frac{\Theta_{\mathrm{D}}}{\mathrm{T}}+\frac{1}{3} \ln \left(1-\mathrm{e}^{-\Theta_{\mathrm{D}} / \mathrm{T}}\right)-\mathrm{D}\left(\Theta_{\mathrm{D}} / \mathrm{T}\right)\right]
$$

where $k_{B}$ is the Boltzmann constant, $n$ is the number of atoms per formula unit, $D\left(\Theta_{D} / T\right)$ is the Debye integral defined as

$$
\mathrm{D}(\mathrm{x})=\frac{3}{\mathrm{x}^{3}} \int_{0}^{\mathrm{x}} \frac{\mathrm{y}^{3}}{\mathrm{e}^{\mathrm{y}}-1} \mathrm{dy}
$$

For an isotropic solid, the Debye temperature $\Theta_{D}$ can be written as:

$$
\Theta_{\mathrm{D}}=\frac{\hbar}{\mathrm{k}_{\mathrm{B}}}\left(6 \pi^{2} \mathrm{~V}^{1 / 2} \mathrm{n}\right)^{1 / 3} \mathrm{f}(\mathrm{v}) \sqrt{\frac{\mathrm{B}_{\mathrm{S}}}{\mathrm{M}}}
$$


where $\mathrm{M}$ is the molecular mass per formula unit and $\mathrm{B}_{\mathrm{S}}$ the static bulk modulus, which can be approximated by the static compressibility:

$$
\mathrm{B}_{\mathrm{S}}=\mathrm{B}_{\text {static }}(\mathrm{V})=\mathrm{V}\left[\frac{\mathrm{d}^{2} \mathrm{E}(\mathrm{V})}{\mathrm{dV^{2 }}}\right]
$$

and $\nu$ is the Poisson's ratio and $f(v)$ is given as:

$$
f(v)=\left\{3\left[2\left(\frac{2}{3} \frac{1+v}{1-2 v}\right)^{3 / 2}+\left(\frac{1}{3} \frac{1+v}{1-v}\right)^{3 / 2}\right]^{-1}\right\}^{1 / 3}
$$

As a result, the non-equilibrium Gibbs functions can be minimized with respect to volume $\mathrm{V}$ as follow:

$$
\left(\frac{\partial \mathrm{G}^{*}(\mathrm{~V} ; \mathrm{P}, \mathrm{T})}{\partial \mathrm{V}}\right)_{\mathrm{P}, \mathrm{T}}=0
$$

Then, the thermal equation of state (EOS) can be obtained by solving the above equation and the isothermal bulk modulus $B_{T}$, the heat capacity $C_{V}$ at constant volume and $C_{P}$ at constant pressure are given by the following equations:

$$
\begin{gathered}
\mathrm{B}_{\mathrm{T}}(\mathrm{P}, \mathrm{T})=\mathrm{V}\left[\frac{\partial^{2} \mathrm{G}^{*}(\mathrm{~V} ; \mathrm{P}, \mathrm{T})}{\partial \mathrm{V}^{2}}\right]_{\mathrm{P}, \mathrm{T}} \\
\mathrm{C}_{\mathrm{V}}=3 \mathrm{nk}_{\mathrm{B}}\left[4 \mathrm{D}\left(\Theta_{\mathrm{D}} / \mathrm{T}\right)-\frac{3 \Theta_{\mathrm{D}} / \mathrm{T}}{\mathrm{e}^{\Theta_{\mathrm{D}} / \mathrm{T}}-1}\right] \\
\mathrm{C}_{\mathrm{P}}=\mathrm{C}_{\mathrm{V}}(1+\alpha \gamma \mathrm{T})
\end{gathered}
$$

where the thermal expansion coefficient $\alpha$ and the Grüneisen constant $\gamma$ are

$$
\alpha=\frac{\gamma C_{V}}{B_{T} V}, \quad \gamma=\frac{d \ln \Theta_{\mathrm{D}}(V)}{d \ln V}
$$

\section{Results and Discussion}

\subsection{Electronic, Magnetic and Half-Metallic Behaviors}

Based on the obtained equilibrium lattice constant at null pressure and the optimized structure at different pressures, the electronic band structure of $\mathrm{Ti}_{2} \mathrm{NiIn}$ compound has been calculated and displayed in Figure 3. The Fermi energy level is represented by the zero energy in the figure. It can be seen clearly that this material exhibits half-metallic behavior because there is no band gap in the majority spin, but a band gap exists in the minority spin. Through the currently applied pressure from 0 to $50 \mathrm{GPa}$, there is always a band gap in the minority spin and the Fermi level is kept inside this gap, meaning the half-metallicity of $\mathrm{Ti}_{2} \mathrm{NiIn}$ compound is maintained even to the high pressure of $50 \mathrm{GPa}$. A closer look at the band structure, especially for the band gap in the minority spin channel, we found that as the $\mathrm{Ti}_{2} \mathrm{NiIn}$ is compressed with increased pressure, the valence band maximum (VBM) in the minority spin is continuously shifted downwards while the conduction band minimum (CBM) is almost unchanged, leading to the increase of the band gap in the minority spin, as shown in Figure 4, which is also confirmed by the total density of state in the figure. For the majority spin, there is only a very small variation of the band structure, especially at the $G$ point. 


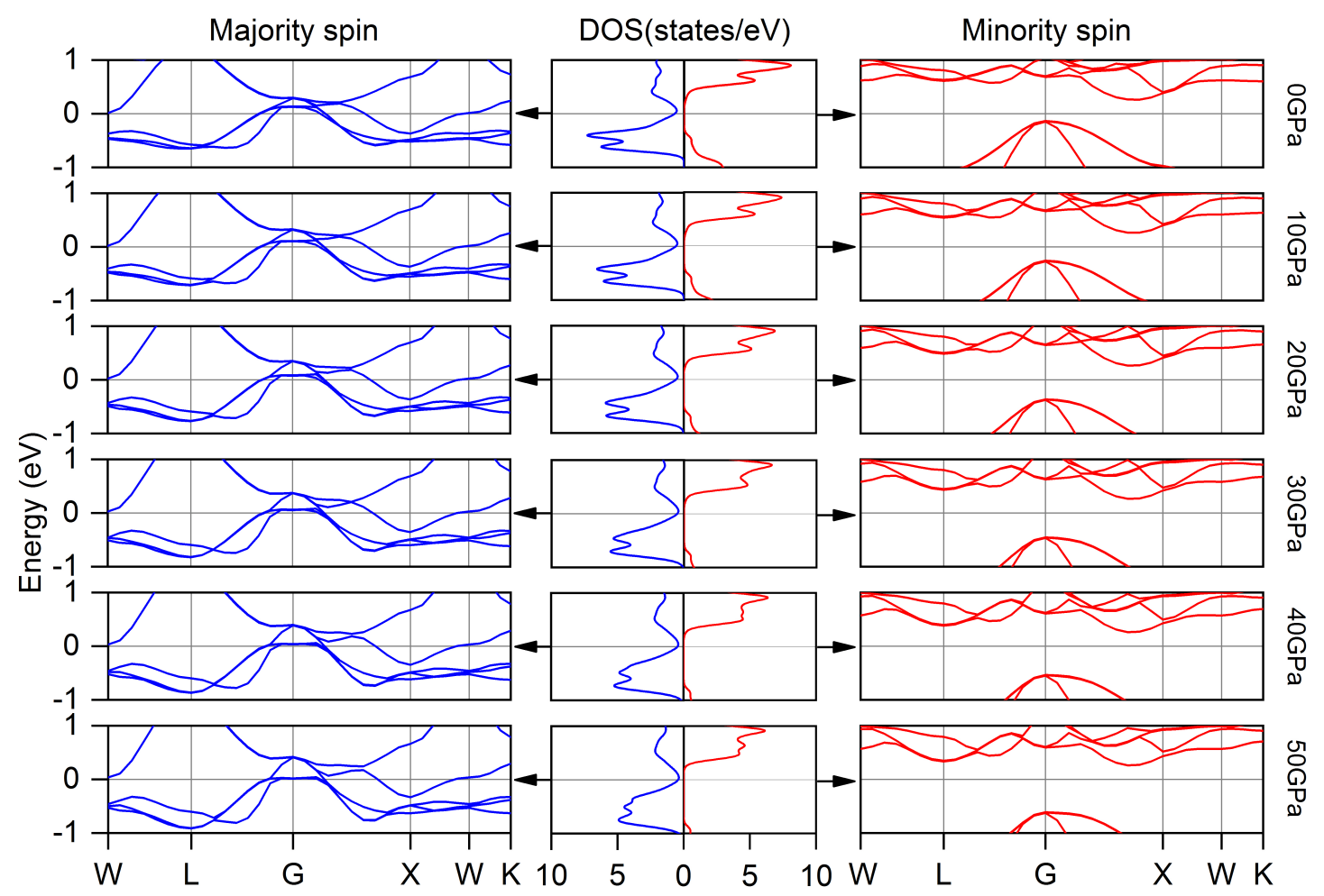

Figure 3. The electronic band structure and total density of states of $\mathrm{Ti}_{2} \mathrm{NiIn}$ compound around the Fermi energy level $(0 \mathrm{eV})$ under different pressure.

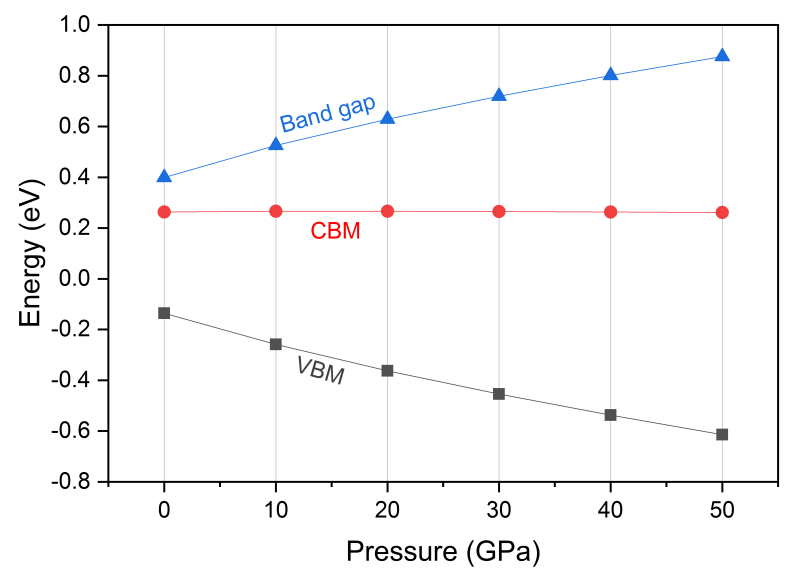

Figure 4. The conduction band minimum (CBM), valence band maximum (VBM) and band gap in the minority spin channel of $\mathrm{Ti}_{2} \mathrm{NiIn}$ compound under different pressure.

The total magnetic moment (TMM) and the atom-resolved spin magnetic moment (AMM) of atom $\mathrm{Ti}, \mathrm{Ni}$ and In under pressure from 0 to $50 \mathrm{GPa}$ are calculated and plotted in Figure 5. The magnetic moments of $\mathrm{Ni}$ and In atoms are always negative and also very small. With pressure increase, their magnetic moments only show a very tiny increase. Therefore, the total magnetic moment is mainly from Ti atoms from both two sites. The two sites of Ti atoms both have positive magnetic moments: for site I, the AMM of Ti does not change with pressure; whereas, for site II, there is a slight decrease along pressure increase. From the different variation effects of AMMs of all atoms, the TMM is always equal to an integer value of $3 \mu_{\mathrm{B}}$ through the whole pressure range. This integral behavior of the TMM is a typical characteristic of the Heusler compound. The $\mathrm{Ti}_{2} \mathrm{NiIn}$ material has 21 valence electrons $\left(\mathrm{Z}_{t}\right)$ and 
the total magnetic moment $\left(\mathrm{M}_{t}\right)$ obeys the Slater-Pauling rule [19,29], $\mathrm{M}_{t}=\mathrm{Z}_{\mathrm{t}}-18$. All these magnetic behaviors show a good stability again pressure variation to the applied pressure limit of $50 \mathrm{GPa}$.

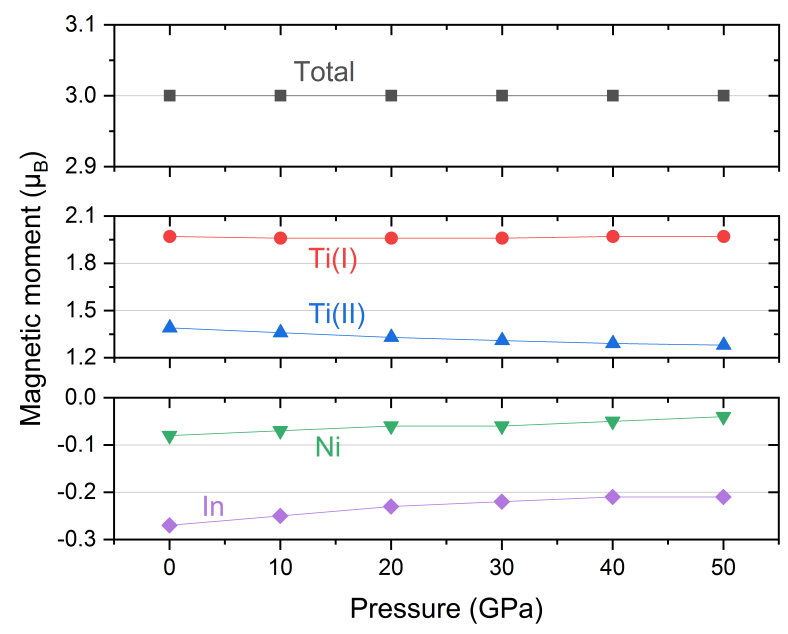

Figure 5. Total and atom-resolved magnetic momentum for $\mathrm{Ti}_{2} \mathrm{NiIn}$ compound under different pressure. Atomic site is referred as the inset crystal structure in Figure 1.

During film growth, this tetragonal distortion often occurs and, then, the physical properties of material may vary greatly. Consequently, we further study the effects of tetragonal distortion on the electronic and magnetic properties of the $\mathrm{Ti}_{2} \mathrm{NiIn}$ compound. By keeping the unit cell volume constantly at the equilibrium condition and varying the ratio of $\mathrm{c} / \mathrm{a}$ from 0.9 to 1.1 , we realize different tetragonal structures as shown in the left panel of Figure 6 and calculate the electronic and magnetic properties of $\mathrm{Ti}_{2} \mathrm{NiIn}$ accordingly. The results are shown in Figures 6 and 7 for the electronic and magnetic behaviors, respectively.
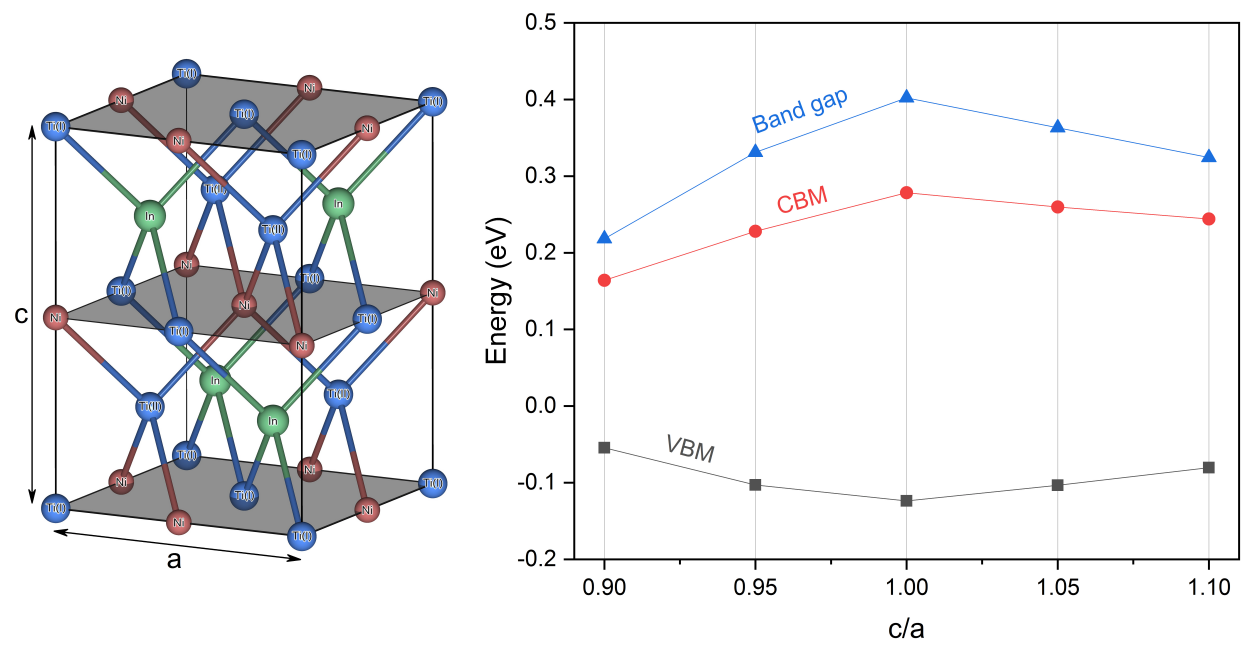

Figure 6. The tetragonal structure and the conduction band minimum (CBM), valence band maximum (VBM) and band gap in the minority spin channel of $\mathrm{Ti}_{2} \mathrm{NiIn}$ compound under tetragonal deformation of different c/a ratio.

It can be seen that with the ratio of $\mathrm{c} / \mathrm{a}$ changing from 1 (no distortion) to either smaller or larger values, the valence band maximum always increases, yet, the conduction band minimum decreases. However, the variation amplitude of the conduction band minimum is larger than that of the valence band maximum, which leads to the band gap decrease at c/a ratio different from 1 . Moreover, we found the effect of $c / a$ ration is a little stronger when it is smaller than one. For the magnetic moment of each 
atom, the effect of $\mathrm{c}$ /a ratio is shown in Figure 7 and there are very small changes only for $\mathrm{Ti}(\mathrm{I})$ and $\mathrm{Ni}$ atoms by the tetragonal distortion. The total magnetic moment keeps the same value of $3 \mu_{\mathrm{B}}$, meaning that the magnetic behavior of $\mathrm{Ti}_{2} \mathrm{NiIn}$ compound shows some resistance to tetragonal distortion.

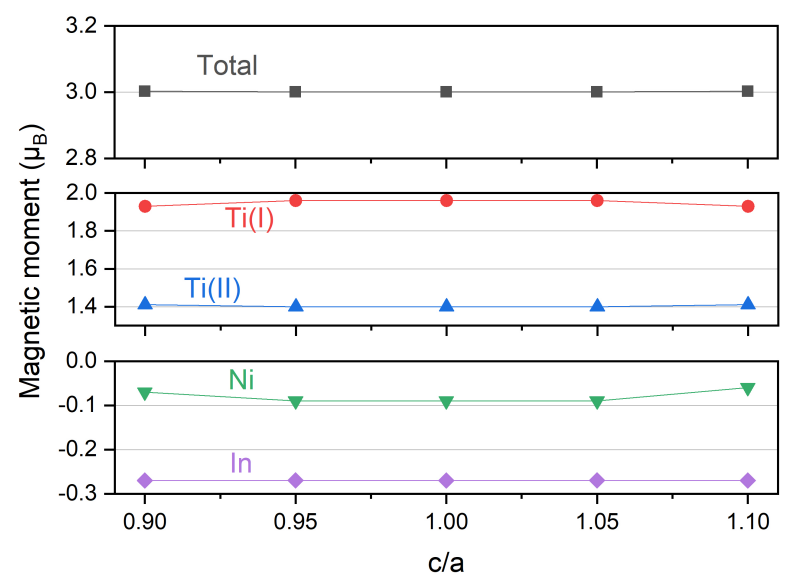

Figure 7. Total and atom-resolved magnetic momentum for $\mathrm{Ti}_{2} \mathrm{NiIn}$ compound under tetragonal deformation of different c/a ratio. Atomic site is referred as the inset crystal structure in Figure 1.

\subsection{Mechanic Properties}

The mechanical property of material plays a critical role for its application. For the studied cubic $\mathrm{Ti}_{2} \mathrm{NiIn}$ compound, three independent elastic constants together with the bulk modulus $\mathrm{B}$, shear modulus G, Young's modulus E and Pugh's ratio B/G are calculated as described in Section 2.3 under the pressure range from 0 to $50 \mathrm{GPa}$. The results are listed in Table 1 and also plotted in Figure 8. The obtained results at low pressure range from 0 to $10 \mathrm{GPa}$, and match other studies very well [27-29,33,34]. From the three elastic constants and bulk modulus, the mechanical stability of $\mathrm{Ti}_{2}$ NiIn compound is examined with the generalized Born-Huang elastic stability criteria $[37,38]$, see Equation (1). It is found that through the whole pressure range from 0 to $50 \mathrm{GPa}$, the $\mathrm{Ti}_{2} \mathrm{NiIn}$ compound satisfies this criteria and thus is mechanically stable. From Figure 8, one can see that all the three elastic constants $C_{i j}$ show a moderate increase with pressure. But, $C_{11}$ and $C_{12}$ have much larger change with pressure than $\mathrm{C}_{44}$. Obviously, the increases of elastic constants and moduli with pressure make the material stronger and more difficult to compress. The Pugh's ratio can be used to predict whether the material is brittle or ductile and it is equal to 2.29 at null pressure, larger than 1.75 , indicating that this compound is ductile. With pressure increase, the Pugh's ratio shows a upward trend, meaning the $\mathrm{Ti}_{2} \mathrm{NiIn}$ compound becomes more and more ductile at higher pressure. From Figure 8 it can also be observed that the bulk modulus B, shear modulus G and Young's modulus E increase monotonically with pressure increase from 0 to $50 \mathrm{GPa}$. However, the variation of $\mathrm{G}$ is smaller than that of $\mathrm{B}$ and $\mathrm{E}$.

Table 1. The elastic constants $C_{\mathrm{ij}}$, bulk modulus B, shear modulus $G$, Young's modulus E and Pugh's ratio $\mathrm{B} / \mathrm{G}$ of $\mathrm{Ti}_{2} \mathrm{NiIn}$ compound under different pressure.

\begin{tabular}{cccccccc}
\hline $\begin{array}{c}\mathbf{P} \\
(\mathbf{G P a})\end{array}$ & $\begin{array}{c}\mathbf{C}_{\mathbf{1 1}} \\
\mathbf{( G P a})\end{array}$ & $\begin{array}{c}\mathbf{C}_{\mathbf{1 2}} \\
\mathbf{( G P a})\end{array}$ & $\begin{array}{c}\mathbf{C}_{\mathbf{4 4}} \\
\mathbf{( G P a})\end{array}$ & $\begin{array}{c}\mathbf{B} \\
\mathbf{G P a})\end{array}$ & $\begin{array}{c}\mathbf{G} \\
\mathbf{G P a})\end{array}$ & $\begin{array}{c}\mathbf{E} \\
(\mathbf{G P a})\end{array}$ & $\mathbf{B} / \mathbf{G}$ \\
\hline 0 & 145.06 & 101.66 & 88.24 & 116.13 & 50.63 & 132.61 & 2.29 \\
10 & 200.41 & 143.84 & 108.47 & 162.70 & 63.61 & 168.83 & 2.56 \\
20 & 246.02 & 177.93 & 126.21 & 200.63 & 74.97 & 199.99 & 2.68 \\
30 & 286.15 & 207.09 & 142.41 & 233.44 & 85.52 & 228.64 & 2.73 \\
40 & 330.37 & 241.47 & 157.26 & 271.10 & 95.09 & 255.40 & 2.85 \\
50 & 372.05 & 273.39 & 163.73 & 306.28 & 101.45 & 274.09 & 3.02 \\
\hline
\end{tabular}




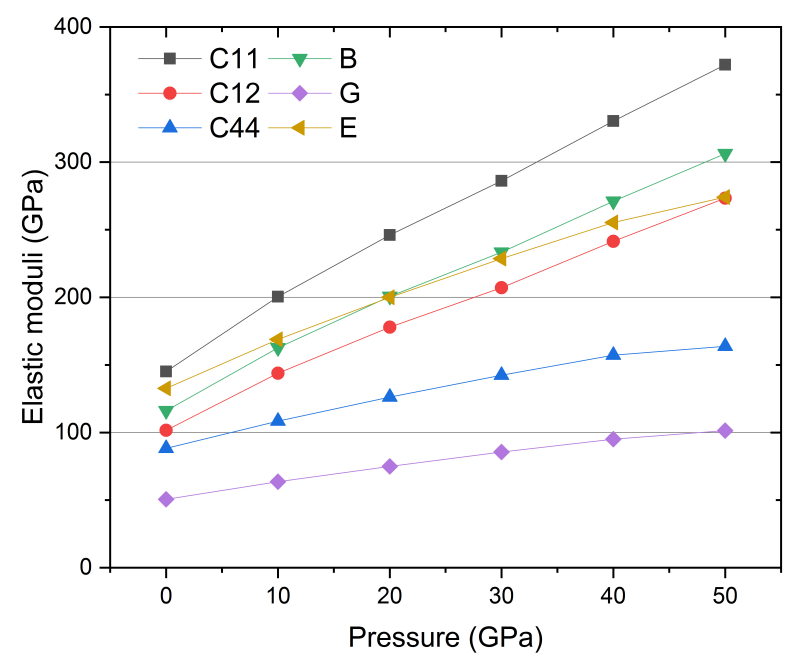

Figure 8. The elastic constants $C_{\mathrm{ij}}$, bulk modulus $\mathrm{B}$, shear modulus $\mathrm{G}$ and Young's modulus $\mathrm{E}$ of $\mathrm{Ti}_{2} \mathrm{NiIn}$ compound under different pressure.

\subsection{Thermodynamic Properties}

The thermodynamic properties can provide us much more information about the material's specific behaviors under severe constraints, such as high temperature or high pressure conditions. Thus, we further investigate the influence of temperature and temperature on the thermodynamic parameters, e.g., thermal expansion coefficient $\alpha$, heat capacity $C_{V}$, Grüneisen constant $\gamma$ and Debye temperature $\Theta_{D}$, by applying the quasi-harmonic Debye model [42-44]. The thermodynamic properties are studied in the temperature range from 0 to $1500 \mathrm{~K}$ under a series of pressure values from 0 to $50 \mathrm{GPa}$.

Figure 9 shows the variation of the normalized unit cell volume $\mathrm{V} / \mathrm{V}_{0}$ under different pressure and temperature values for $\mathrm{Ti}_{2} \mathrm{NiIn}$, where $\mathrm{V}$ is the volume at pressure $\mathrm{P}$ and temperature $\mathrm{T}$ and $\mathrm{V}_{0}$ is the equilibrium volume at null pressure and $0 \mathrm{~K}$. It clearly shows that the relative volume increases with increasing temperature for all the pressure series, see the left panel of Figure 9. This result is apparently expected because commonly material expands at higher temperature. Besides, the volume variation with temperature at lower pressure is stronger than at higher pressure. With pressure increase, the relative volume simply decreases for all different temperature series because material is compressed at higher pressure. Moreover, the volume at higher temperature shows larger variation with pressure than that at lower temperature. Furthermore, we found that the volume is more sensitive to pressure than temperature, i.e., the volume variation with pressure is more important than that of temperature.

The thermal expansion coefficient $\alpha$ has both theoretical and practical significances and also it is essential to predicate the thermodynamic equation of state. The variation of $\alpha$ with temperature and pressure is displayed in Figure 10. It can be seen that the thermal expansion $\alpha$ increases markedly with temperature from 0 to $1500 \mathrm{~K}$; also, the increasing rate is much larger at initial temperature increase from 0 to $300 \mathrm{~K}$. This is because the anharmonic effect of the Debye model at lower temperature is strongly depressed at high temperature. The variation of $\alpha$ with temperature is stronger at lower pressure. From the right panel of Figure 10, we found the $\alpha$ decreases continuously with pressure increase and also the variation trend is stronger at the initial pressure change from 0 to $10 \mathrm{GPa}$, especially for the higher temperature series. At null pressure and $300 \mathrm{~K}$, the thermal expansion $\alpha$ for the studied $\mathrm{Ti}_{2} \mathrm{NiIn}$ compound is $4.34 \times 10^{-5} \mathrm{~K}^{-1}$. 

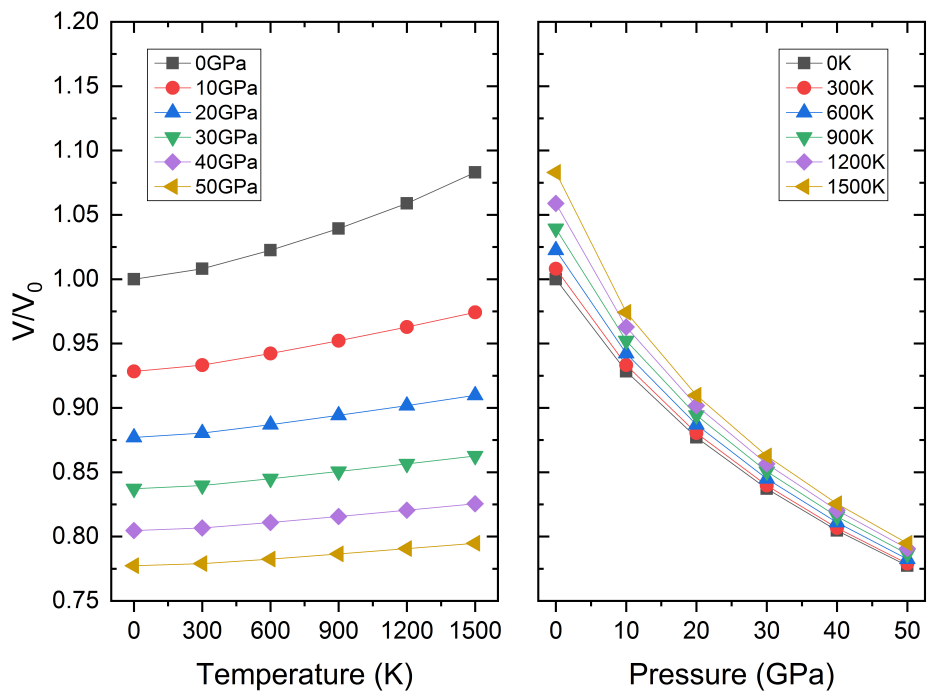

Figure 9. The normalized volume $\mathrm{V} / \mathrm{V}_{0}$ variation with temperature (left) and pressure (right) for $\mathrm{Ti}_{2} \mathrm{NiIn}$.
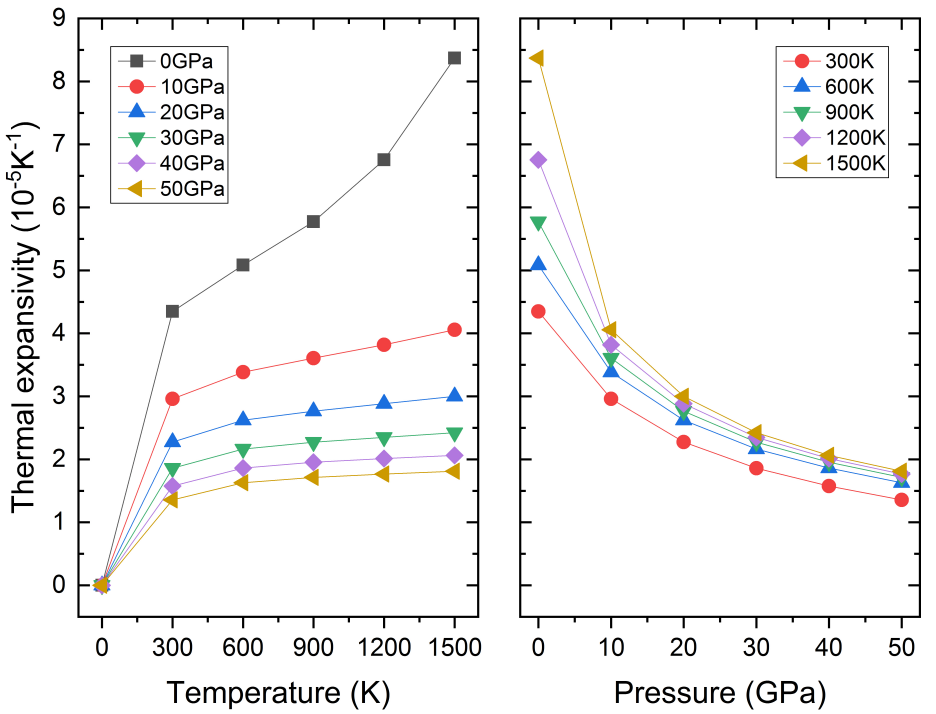

Figure 10. The thermal expansion coefficient $\alpha$ variation with temperature (left) and pressure (right) for $\mathrm{Ti}_{2} \mathrm{NiIn}$.

Next, we examine the heat capacity $C_{V}$ and $C_{P}$ of $T_{2}$ NiIn dependence on temperature and pressure. Commonly, heat capacity is a critical parameter for the material physical properties and it can provide significant information about the lattice vibration and transition of phase. The result is plotted in Figure 11. It is found that the temperature has a very strong effect at the low temperature from 0 to $300 \mathrm{~K}$ because of the sharp increase of $C_{V}$ and $C_{P}$. This is because of the anharmonic approximation of the Debye model at lower temperature. Further increasing temperature, this anharmonic part is suppressed and $C_{V}$ saturates quickly to the Dulong-Petit limit, which indicates that thermal energy excites all the possible phonon mode at higher temperature and is common to all solids. While for $C_{P}$, it continues to increase slowly with temperature and this different behaviors can be easily understood by Equation 14. Besides, the pressure has reverse impact, i.e., both $C_{V}$ and $C_{P}$ decrease with pressure increase. The changing rate is reduced with temperature increase. At zero pressure and $300 \mathrm{~K}$, the $\mathrm{C}_{V}$ and $\mathrm{C}_{\mathrm{P}}$ for the studied $\mathrm{Ti}_{2} \mathrm{NiIn}$ compound is equal to $91.3 \mathrm{JMol}^{-1} \mathrm{~K}^{-1}$ and $93.7 \mathrm{JMol}^{-1} \mathrm{~K}^{-1}$. 

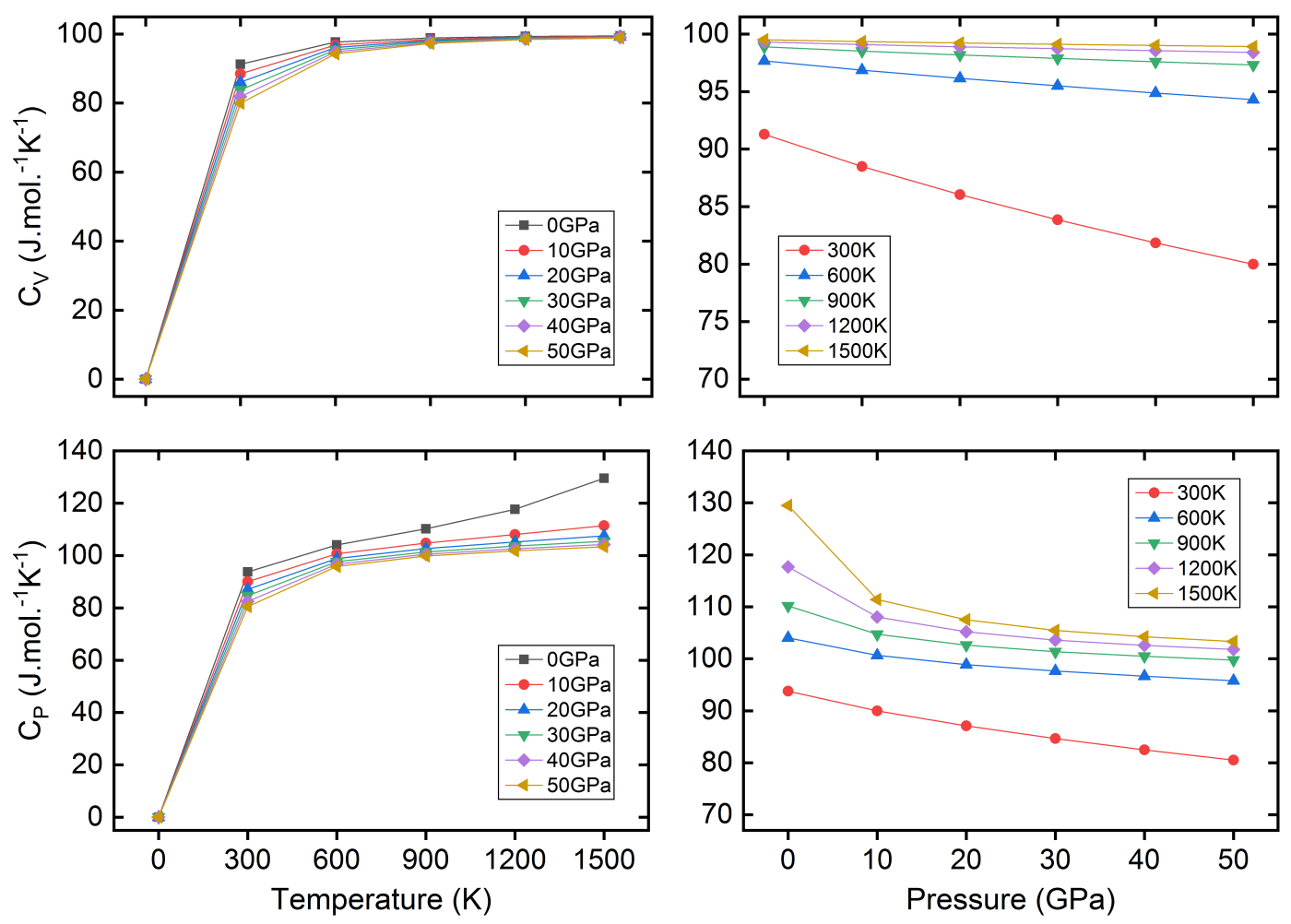

Figure 11. The variation of heat capacity $C_{V}$ and $C_{P}$ with temperature (left) and pressure (right) for $\mathrm{Ti}_{2} \mathrm{NiIn}$.

The Grüneisen constant $\gamma$ is of great interest because it appears in some important thermodynamic relations. The dependence of $\gamma$ on temperature and pressure is calculated and plotted in Figure 12. With increasing temperature, Grüneisen constant $\gamma$ continuously increases and its amplitude of variation from 0 to $1500 \mathrm{~K}$ is strongly suppressed by higher pressure. Moreover, the increasing rate of $\gamma$ with temperature is bigger at higher temperature; though, there is very small change between 0 and $300 \mathrm{~K}$. For a given temperature, $\gamma$ decreases with pressure increase. The calculated $\gamma$ of $\mathrm{Ti}_{2} \mathrm{NiIn}$ compound at zero pressure and $300 \mathrm{~K}$ is 2.085 .

Finally, we investigate the effect of temperature and pressure on the Debye temperature $\Theta_{D}$ and the result is shown in Figure 13. When the temperature of the material is below its Debye temperature, the lattice vibration is mainly excited from acoustic vibration and the quantum mechanical effect plays a critical role in the thermodynamic properties. However, these can be negligible when the temperature is above the Debye temperature. It can be seen in Figure 13, that $\Theta_{\mathrm{D}}$ smoothly decreases with increasing temperature and the variation trend is larger at lower pressure. Furthermore, the decreasing rate of $\Theta_{\mathrm{D}}$ with temperature becomes stronger at higher temperature and there is a very small variation at low temperature from 0 to $300 \mathrm{~K}$. However, with pressure increase, $\Theta_{\mathrm{D}}$ continuously increases. The calculated $\Theta_{\mathrm{D}}$ of the studied $\mathrm{Ti}_{2} \mathrm{NiIn}$ compound at zero pressure and $300 \mathrm{~K}$ is $403.88 \mathrm{~K}$. 

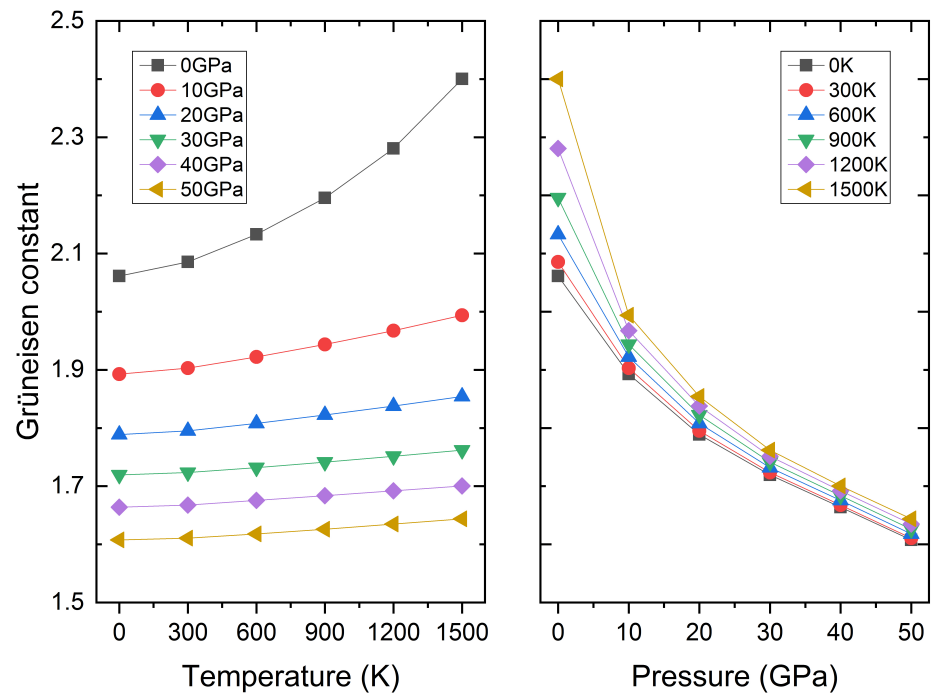

Figure 12. The Grüneisen constant $\gamma$ variation with temperature (left) and pressure (right) for $\mathrm{Ti}_{2} \mathrm{NiIn}$.
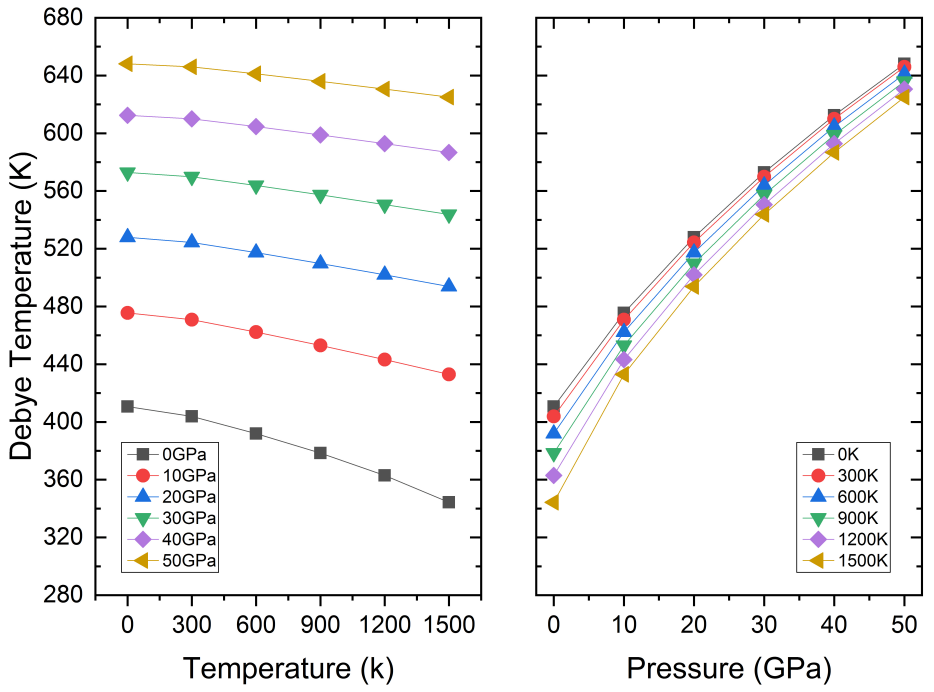

Figure 13. The Debye temperature $\Theta_{\mathrm{D}}$ variation with temperature (left) and pressure (right) for $\mathrm{Ti}_{2} \mathrm{NiIn}$.

\section{Conclusions}

In this work, we employ first-principles calculations based on density functional theory and systematically study the structural, electronic, magnetic and mechanical properties of the inverse Heusler compound $\mathrm{Ti}_{2} \mathrm{NiIn}$ under a series of external pressure. The obtained equilibrium lattice constant and electronic band structure at null pressure are consistent with previous studies. By applying static pressure from 0 to $50 \mathrm{GPa}$, this material maintains its half-metallicity and also its total magnetic moment is kept the same as $3 \mu_{\mathrm{B}}$, which obeys the Slater-Pauling rule. This means the $\mathrm{Ti}_{2} \mathrm{NiIn}$ is robust against pressure changes. Along the pressure increase, all the elastic constants and moduli monotonically increase. With the generalized elastic stability criteria, $\mathrm{Ti}_{2} \mathrm{NiIn}$ is mechanically stable through the studied pressure range. The Pugh's ratio of $\mathrm{Ti}_{2} \mathrm{NiIn}$ at null pressure is 2.29 and increases with increasing pressure, reflecting its stronger ductility. Furthermore, we examine the thermodynamic properties of this material by applying the quasi-harmonic Debye model and obtain the dependence on temperature and pressure of several parameters including the normalized volume $\mathrm{V} / \mathrm{V}_{0}$, thermal expansion coefficient $\alpha$, heat capacity $C_{V}$, Grüneisen constant $\gamma$ and Debye temperature $\Theta_{D}$. It is found that $\alpha, \gamma$ and $C_{V}$ increase with increasing temperature and decrease with increasing 
pressure, while an opposite effect of temperature and pressure on $\Theta_{\mathrm{D}}$ is seen. For different parameters, the amplitude of variation from temperature and pressure show different trends. This theoretical study can provide detailed information and valuable reference for further experimental work.

Author Contributions: T.Y. conceived the work and J.C. performed the calculations; T.Y, and J.C. wrote the paper; X.W. supervised this work; X.W. and J.C. provided valuable comments on the manuscript.

Funding: This research was supported by "Fundamental Research Funds for the Central Universities" grant number [XDJK2018C078] and "Doctoral Fund Project of Southwest University" grant number [SWU117037].

Conflicts of Interest: The authors declare no conflict of interest.

\section{References}

1. Žutić, I.; Fabian, J.; Das Sarma, S. Spintronics: Fundamentals and applications. Rev. Mod. Phys. 2004, 76, 323-410. [CrossRef]

2. de Groot, R.A.; Mueller, F.M.; van Engen, P.G.; Buschow, K.H.J. New Class of Materials: Half-Metallic Ferromagnets. Phys. Rev. Lett. 1983, 50, 2024-2027. [CrossRef]

3. van Leuken, H.; de Groot, R.A. Half-Metallic Antiferromagnets. Phys. Rev. Lett. 1995, 74, 1171-1173. [CrossRef] [PubMed]

4. Gao, G.Y.; Hu, L.; Yao, K.L.; Luo, B.; Liu, N. Large half-metallic gaps in the quaternary Heusler alloys $\mathrm{CoFeCrZ} \mathrm{(Z=Al,} \mathrm{Si,} \mathrm{Ga,} \mathrm{Ge):} \mathrm{A} \mathrm{first-principles} \mathrm{study.} \mathrm{J.} \mathrm{Alloys} \mathrm{Compd.} \mathrm{2013,} \mathrm{551,} \mathrm{539-543.} \mathrm{[CrossRef]}$

5. Han, Y.; Wu, Y.; Li, T.; Khenata, R.; Yang, T.; Wang, X. Electronic, Magnetic, Half-Metallic, and Mechanical Properties of a New Equiatomic Quaternary Heusler Compound YRhTiGe: A First-Principles Study. Materials 2018, 11, 797. [CrossRef] [PubMed]

6. Alijani, V.; Ouardi, S.; Fecher, G.H.; Winterlik, J.; Naghavi, S.S.; Kozina, X.; Stryganyuk, G.; Felser, C.; Ikenaga, E.; Yamashita, Y.; et al. Electronic, structural, and magnetic properties of the half-metallic ferromagnetic quaternary Heusler compounds CoFeMnZ ( $\mathrm{Z}=\mathrm{Al}, \mathrm{Ga}, \mathrm{Si}, \mathrm{Ge})$. Phys. Rev. B 2011, 84, 224416. [CrossRef]

7. Bainsla, L.; Mallick, A.I.; Raja, M.M.; Nigam, A.K.; Varaprasad, B.S.D.C.S.; Takahashi, Y.K.; Alam, A.; Suresh, K.G.; Hono, K. Spin gapless semiconducting behavior in equiatomic quaternary CoFeMnSi Heusler alloy. Phys. Rev. B 2015, 91, 104408. [CrossRef]

8. Wang, X.; Khachai, H.; Khenata, R.; Yuan, H.; Wang, L.; Wang, W.; Bouhemadou, A.; Hao, L.; Dai, X.; Guo, R.; et al. Structural, electronic, magnetic, half-metallic, mechanical, and thermodynamic properties of the quaternary Heusler compound FeCrRuSi: A first-principles study. Sci. Rep. 2017, 7, 16183. [CrossRef] [PubMed]

9. Bainsla, L.; Suresh, K.G. Equiatomic quaternary Heusler alloys: A material perspective for spintronic applications. Appl. Phys. Rev. 2016, 3, 031101. [CrossRef]

10. Bainsla, L.; Mallick, A.I.; Coelho, A.A.; Nigam, A.K.; Varaprasad, B.S.D.C.S.; Takahashi, Y.K.; Alam, A.; Suresh, K.G.; Hono, K. High spin polarization and spin splitting in equiatomic quaternary CoFeCrAl Heusler alloy. J. Magn. Magn. Mater. 2015, 394, 82-86. [CrossRef]

11. Feng, Y.; Chen, X.; Zhou, T.; Yuan, H.; Chen, H. Structural stability, half-metallicity and magnetism of the CoFeMnSi/GaAs(001) interface. Appl. Surf. Sci. 2015, 346, 1-10. [CrossRef]

12. Wurmehl, S.; Fecher, G.H.; Kandpal, H.C.; Ksenofontov, V.; Felser, C.; Lin, H.J. Investigation of Co2FeSi: The Heusler compound with highest Curie temperature and magnetic moment. Appl. Phys. Lett. 2006, 88, 032503. [CrossRef]

13. Gofryk, K.; Kaczorowski, D.; Plackowski, T.; Leithe-Jasper, A.; Grin, Y. Magnetic and transport properties of rare-earth-based half-Heusler phases RPdBi: Prospective systems for topological quantum phenomena. Phys. Rev. B 2011, 84, 035208. [CrossRef]

14. Nayak, A.K.; Suresh, K.G.; Nigam, A.K. Anomalous effects of repeated martensitic transitions on the transport, magnetic and thermal properties in Ni-Co-Mn-Sb Heusler alloy. Acta Mater. 2011, 59, 3304-3312. [CrossRef]

15. Wang, X.T.; Cui, Y.T.; Liu, X.F.; Liu, G.D. Electronic structures and magnetism in the $\mathrm{Li}_{2} \mathrm{AgSb}$-type Heusler alloys, $\mathrm{Zr}_{2} \mathrm{CoZ}$ (Z=Al, Ga, In, Si, Ge, Sn, Pb, Sb): A first-principles study. J. Magn. Magn. Mater. 2015, 394, 50-59. [CrossRef] 
16. Babiker A, S.; Gao, G.; Yao, K. Half-metallicity and magnetism of Heusler alloys $\mathrm{Co}_{2} \mathrm{HfZ}$ (Z=Al, Ga, Ge, Sn). J. Magn. Magn. Mater. 2017, 441, 356-360. [CrossRef]

17. Akriche, A.; Bouafia, H.; Hiadsi, S.; Abidri, B.; Sahli, B.; Elchikh, M.; Timaoui, M.A.; Djebour, B. First-principles study of mechanical, exchange interactions and the robustness in $\mathrm{Co}_{2} \mathrm{MnSi}$ full Heusler compounds. J. Magn. Magn. Mater. 2017, 422, 13-19. [CrossRef]

18. Wang, L.; Jin, Y. A spin-gapless semiconductor of inverse Heusler $\mathrm{Ti}_{2} \mathrm{CrSi}$ alloy: First-principles prediction. J. Magn. Magn. Mater. 2015, 385, 55-59. [CrossRef]

19. Wei, X.P.; Deng, J.B.; Mao, G.Y.; Chu, S.B.; Hu, X.R. Half-metallic properties for the $\mathrm{Ti}_{2} \mathrm{YZ}(\mathrm{Y}=\mathrm{Fe}, \mathrm{Co}, \mathrm{Ni}, \mathrm{Z}$ $=\mathrm{Al}, \mathrm{Ga}$, In) Heusler alloys: A first-principles study. Intermetallics 2012, 29, 86-91. [CrossRef]

20. Zheng, N.; Jin, Y. Band-gap and Slater-Pauling rule in half-metallic $\mathrm{Ti}_{2}$-based Heusler alloys: A first-principles study. J. Magn. Magn. Mater. 2012, 324, 3099-3104. [CrossRef]

21. Birsan, A.; Palade, P.; Kuncser, V. Half-metallic state and magnetic properties versus the lattice constant in $\mathrm{Ti}_{2} \mathrm{CoSn}$ Heusler compound: An ab initio study. Solid State Commun. 2012, 152, 2147-2150. [CrossRef]

22. Fang, Q.L.; Zhang, J.M.; Xu, K.W. Magnetic properties and origin of the half-metallicity of $\mathrm{Ti}_{2} \mathrm{MnZ}(\mathrm{Z}=\mathrm{Al}$, $\mathrm{Ga}$, In, Si, Ge, Sn) Heusler alloys with the $\mathrm{Hg}_{2} \mathrm{CuTi-type} \mathrm{structure.} \mathrm{J.} \mathrm{Magn.} \mathrm{Magn.} \mathrm{Mater.} \mathrm{2014,} \mathrm{349,} 104-108$. [CrossRef]

23. Yang, T.; Hao, L.; Khenata, R.; Wang, X. Strain Conditions for the Inverse Heusler Type Fully Compensated Spin-Gapless Semiconductor $\mathrm{Ti}_{2} \mathrm{MnAl}$ : A First-Principles Study. Materials 2018, 11, 2091. [CrossRef] [PubMed]

24. Jia, H.Y.; Dai, X.F.; Wang, L.Y.; Liu, R.; Wang, X.T.; Li, P.P.; Cui, Y.T.; Liu, G.D. Ti ${ }_{2} \mathrm{MnZ}$ (Z = Al, Ga, In) compounds: Nearly spin gapless semiconductors. AIP Adv. 2014, 4, 047113. [CrossRef]

25. Shi, W.; Muechler, L.; Manna, K.; Zhang, Y.; Koepernik, K.; Car, R.; van den Brink, J.; Felser, C.; Sun, Y. Prediction of a magnetic Weyl semimetal without spin-orbit coupling and strong anomalous Hall effect in the Heusler compensated ferrimagnet $\mathrm{Ti}_{2} \mathrm{MnAl}$. Phys. Rev. B 2018, 97, 060406. [CrossRef]

26. Lei, F.; Tang, C.; Wang, S.; He, W. Half-metallic full-Heusler compound $\mathrm{Ti}_{2} \mathrm{NiAl}$ : A first-principles study. J. Alloys Compd. 2011, 509, 5187-5189. [CrossRef]

27. Feng, L.; Wang, F.; Li, J.; Wang, S.J. A New Ti-Based Half-Metallic Compound: A First-Principles Study. Appl. Mech. Mater. 2013, 320, 399-402. [CrossRef]

28. Boudali, A.; Zemouli, M.; Saadaoui, F.; Khodja, M.D. Structural, Elastic, Electronic, and Magnetic Properties of the Full-Heusler Compounds $\mathrm{Ti}_{2} \mathrm{NiX}(\mathrm{X}=\mathrm{Al}$, Ga, and In). J. Supercond. Novel Magn. 2017, 30, 15-23. [CrossRef]

29. Wei, X.P.; Zhang, Y.L.; Sun, X.W.; Song, T.; Guo, P. The electronic and magnetic properties of defects on half-metallic $\mathrm{Ti}_{2}$ NiIn alloy. J. Solid State Chem. 2016, 233, 221-228. [CrossRef]

30. Payne, M.C.; Teter, M.P.; Allan, D.C.; Arias, T.A.; Joannopoulos, J.D. Iterative minimization techniques for ab initio total-energy calculations: Molecular dynamics and conjugate gradients. Rev. Mod. Phys. 1992, 64, 1045-1097. [CrossRef]

31. Segall, M.D.; Philip, J.D.L.; Probert, M.J.; Pickard, C.J.; Hasnip, P.J.; Clark, S.J.; Payne, M.C. First-principles simulation: Ideas, illustrations and the CASTEP code. J. Phys. Condens. Matter 2002, 14, 2717. [CrossRef]

32. Perdew, J.P.; Burke, K.; Ernzerhof, M. Generalized Gradient Approximation Made Simple. Phys. Rev. Lett. 1996, 77, 3865-3868. [CrossRef] [PubMed]

33. Wen, Y.; Yu, X.; Zeng, X.; Ye, Y.; Wu, D.; Gou, Q. Ab initio calculations of the mechanical and acoustic properties of $\mathrm{Ti}_{2}$-based Heusler alloys under pressures. Eur. Phys. J. B 2018, 91, 140. [CrossRef]

34. Wei, X.P.; Chu, Y.D.; Sun, X.W.; Deng, J.B. First-principles study on stability, electronic and thermodynamic properties of $\mathrm{Ti}_{2} \mathrm{CoIn}$ and $\mathrm{Ti}_{2} \mathrm{NiIn}$. Eur. Phys. J. B 2013, 86, 450. [CrossRef]

35. Pfrommer, B.G.; Côté, M.; Louie, S.G.; Cohen, M.L. Relaxation of Crystals with the Quasi-Newton Method. J. Comput. Phys. 1997, 131, 233-240. [CrossRef]

36. Wang, J.; Li, J.; Yip, S.; Phillpot, S.; Wolf, D. Mechanical instabilities of homogeneous crystals. Phys. Rev. B 1995, 52, 12627-12635. [CrossRef]

37. Sin'ko, G.V.; Smirnov, N.A. Ab initio calculations of elastic constants and thermodynamic properties of bcc, fcc, and hcp Al crystals under pressure. J. Phys. Condens. Matter 2002, 14, 6989.

38. Yip, S.; Li, J.; Tang, M.; Wang, J. Mechanistic aspects and atomic-level consequences of elastic instabilities in homogeneous crystals. Mater. Sci. Eng. A 2001, 317, 236-240. [CrossRef]

39. Hill, R. The Elastic Behaviour of a Crystalline Aggregate. Proc. Phys. Soc. Sect. A 1952, 65, 349. [CrossRef] 
40. Wu, Z.J.; Zhao, E.J.; Xiang, H.P.; Hao, X.F.; Liu, X.J.; Meng, J. Crystal structures and elastic properties of superhard $\mathrm{IrN}_{2}$ and $\mathrm{IrN}_{3}$ from first principles. Phys. Rev. B 2007, 76, 054115. [CrossRef]

41. Cherid, S.; Benstaali, W.; Abbad, A.; Bentata, S.; Lantri, T.; Abbar, B. Theoretical prediction of half metallic ferromagnetic full-Heusler alloys $\mathrm{Cs}_{2} \mathrm{CrGe}$. Solid State Commun. 2017, 260, 14-18. [CrossRef]

42. Blanco, M.A.; Francisco, E.; Luaña, V. GIBBS: Isothermal-isobaric thermodynamics of solids from energy curves using a quasi-harmonic Debye model. Comput. Phys. Commun. 2004, 158, 57-72. [CrossRef]

43. Otero-de-la Roza, A.; Abbasi-Pérez, D.; Luaña, V. Gibbs2: A new version of the quasiharmonic model code. II. Models for solid-state thermodynamics, features and implementation. Comput. Phys. Commun. 2011, 182, 2232-2248. [CrossRef]

44. Otero-de-la Roza, A.; Luaña, V. Gibbs2: A new version of the quasi-harmonic model code. I. Robust treatment of the static data. Comput. Phys. Commun. 2011, 182, 1708-1720. [CrossRef]

2018 by the authors. Licensee MDPI, Basel, Switzerland. This article is an open access article distributed under the terms and conditions of the Creative Commons Attribution (CC BY) license (http://creativecommons.org/licenses/by/4.0/). 